\title{
Lz-Transform Method and Markov Reward Approach for Flexible Manufacturing System Performance Evaluation
}

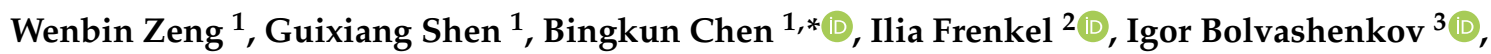 \\ Jörg Kammermann ${ }^{3}{ }^{(}$, Hans-Georg Herzog ${ }^{3}{ }^{-}$, Lev Khvatskin ${ }^{2}$ and Anatoly Lisnianski ${ }^{4}$ \\ 1 Key Laboratory of CNC Equipment Reliability, Ministry of Education, School of Mechanical and Aerospace \\ Engineering, Jilin University, Changchun 130022, China; zengwb15@yeah.net (W.Z.); \\ shengx@jlu.edu.cn (G.S.) \\ 2 Center for Reliability and Risk Management, Shamoon College of Engineering, Beer Sheva 84100, Israel; \\ iliaf@frenkel-online.com (I.F.); khvatskin@gmail.com (L.K.) \\ 3 Institute of Energy Conversion Technology, Technical University of Munich (TUM), 80333 Munich, Germany; \\ igor.bolvashenkov@tum.de (I.B.); joerg.kammermann@tum.de (J.K.); hg.herzog@tum.de (H.-G.H.) \\ 4 The Israel Electric Corporation Ltd., Haifa 31000, Israel; lisnianski@bezeqint.net \\ * Correspondence: chenbk@jlu.edu.cn
}

Received: 1 August 2019; Accepted: 20 September 2019; Published: 3 October 2019

Featured Application: Smart factory, multi-state system, reliability engineering.

\begin{abstract}
This article presents a performance evaluation of a flexible manufacturing system (FMS), in which the system and its components can have different performance levels ranging from perfect functioning to total failure. An original multistate transition process for the FMS's main component, Computerized Numerical Control (CNC) machine tools, proposed in the current article and treated as an aging process because of its increasing failure rate is investigated. The Markov reward approach is utilized to analyze the availability of CNC machine tools and sensitivity analysis of each non-operational state to the machine's downtime. To overcome the "state explosion problem" in the subsequent FMS performance evaluation process, an application of the Lz-transform method is proposed to determine the performance evaluation and availability of such aging multistate FMS. An illustrated case is presented to demonstrate the performance evaluation process. The results show that the suggested methods can be implemented in engineering decision making and construction of various aging multistate FMS related to demands, availability, and output performance.
\end{abstract}

Keywords: Lz-transform method; Markov reward approach; aging multistate CNC machine tools; flexible manufacturing system; availability; output performance

\section{Introduction}

Fierce competition in the market of the manufacturing sector and various customer preferences constantly challenge the diversity of manufacturing firms' products and their resilience to rapid technological change and to shorten the product life cycle. The flexible manufacturing systems (FMS) have, therefore, become an inevitable choice for many companies and a research hotspot [1]. Moreover, the concept of Industry 4.0, such as digitalization, Internet of Things (IoT), and cyber physical systems (CPS) has gained great importance across industries, including manufacturing recently, which require more digitized systems and network integration via smart systems $[2,3]$. Therefore, FMS becomes a key topic and plays an important role in the realization of the Industry 4.0 because it can achieve the goals of Industry 4.0 that include a higher level of operational efficiency 
and productivity, as well as a higher level of automatization [4,5]. An FMS is generally defined as an integrated computer-controlled system with a set of workstations, interconnected by an automated material handling system (MHS) and which can simultaneously perform several different tasks $[6,7]$. It is learned from the literature that the workstations (such as CNC machine tools, industrial robots, washing and measuring machines) are processing stations of FMS, which are involved in performing operations on the part types [8]. The automated material handling system is used for transferring parts from one station to another, while the central computer is used for controlling and coordinating the performance of the workstation and material handling system. As the distinguishing attribute of an FMS, flexibility is regarded as the ability of a manufacturing system to withstand a certain level of variations in partial styles without any interruption in the production line [9]. Manufacturing system flexibility consists of volume flexibility, product flexibility, production flexibility, and delivery flexibility, etc. There is already significant research on these flexibilities features under a crucial assumption that elements of the FMS are available at all times.

Rao [10] combined the diagram and matrix methods to rank different FMS alternatives for the final decision making. Chatterjee and Chakraborty [11] used the six most potential preference-ranking methods for selecting the best FMS for a given manufacturing organization. Taha and Rostam [12] proposed a decision support system to select the optimal alternative CNC machine tool to meet the desired criteria of FMS. Recently, more and more researchers use simulation methods for FMS performance analysis. Singholi et al. [13] presented a framework based on the Taguchi experimental design for evaluating the effects of varying levels of machine and routing flexibility on the makespan, average waiting time (AWT), and average utilization of an FMS. Dosdogru et al. [14] utilized an integrated genetic algorithm, the Monte Carlo method, to solve the stochastic flexible job shop-scheduling problem and to measure the impact of routing flexibility on shop performance. Jain and Raj [15] performed three different approaches to analyze the intensity of performance variables in an organization and proposed the FMS performance index to intensify the factors, which affect FMS. In a multistage production system, Gyulai et al. [16] proposed a simulation-based optimization method that utilizes lower-level shop floor data to calculate robust production plans for final assembly lines of a flexible, multistage production system. Rybicka, J. et al. [17] demonstrated how discrete event simulation (DES) can address complexity in an FMS to optimize the production line performance.

However, the reliability and availability of machines play an important role in the actual performance of FMS. Machine failures cause the greatest impact on the due date and other performance criteria even if there is the option of rerouting the parts to alternative workstations. Machines are a major component of FMS, and often it is not possible to handle machine breakdowns as quickly as the production requirements dictate. In addition, the disturbances caused by these breakdowns lead to scheduling problems, which decrease the productivity of the entire manufacturing operations. This issue points out an important need for the consideration of machine reliability in the performance evaluation process of FMS, especially in light of the increasing complexity of such systems in recent years. Consequently, many researchers have realized the importance of reliability features of the FMS and much work has been contributed.

Das et al. [18] proposed an approach that provides a flexible routing which ensures high overall performance of the cellular manufacturing system (CMS) by minimizing the impact of machine failure through the provision of alternative process routes in case of any machine failure. Elleuch et al. [19] proposed a Markov-based model for reducing the severity of breakdowns and improving performances of the CMS with unreliable machines. Loganathan et al. [20] suggested a methodology for availability evaluation of manufacturing systems using semi-Markov model, which considers variable failure or repair rates. Tan and Gershwin [21] proposed a tool for performance evaluation of general Markovian continuous material flow of two-processing stage production systems with an intermediate finite buffer. Koulamas [22] developed a semi-Markov model to study the effects of tool failures on the performance of a flexible manufacturing cell (FMC). Savsar [23] then developed stochastic models to determine the performance of an FMC under random operational conditions, including random 
failures of cell components (machine tool and robot) in addition to random processing times, random machine loading and unloading times, and random pallet transfer times. Duan et al. [24] put forward a reliability modeling and evaluating methodology for a repairable, non-series, multistate FMS with finite buffers by using an improved vector universal generation function to satisfy the market demands on capacity and capability of the system. Chen et al. [25] proposed a process performance evaluation chart to examine the manufacturing performance of bearing connectors, which can also be used for multiple evaluations of manufacturing performance in other manufacturing industries. Kim and Hwangbo [26] investigated the innovation process for smart manufacturing in the domain of synthetic rubber and its vulcanization process, as well as a real-time sensing technology to the realization of a smart manufacturing environment by building cloud-based infrastructure and models for the pre-detection of defects. More studies about availability analysis of manufacturing systems can be found in [27-31].

Although the aforementioned studies have been undertaken, there are very few studies, which consider the influence of production setup activities on availability, and products' output performance of FMS. Moreover, the procedure for overcoming the "state explosion problem" in the performance evaluation process of FMS has not been found. Therefore, a system-level FMS performance evaluation method based on the Markov reward approach and Lz-transform method is proposed in the current article to analyze the output performance of different products and availability of the whole FMS, which includes various aging multistate CNC machine tools and non-always available MHS. Section 2 makes a brief introduction of the Markov reward approach and Lz-transform method. An original multistate transition process of $\mathrm{CNC}$ machine tools that considers the production flexibility and the performance evaluation procedures of an FMS is presented in Section 3. Numerical results are shown and discussed in Section 4. Finally, concluding remarks and the application of the proposed method are presented.

\section{Brief Description of the Lz-Transform Method and Markov Reward Approach}

The Lz-transform and Markov reward approach have been proven to be a successful and efficient application to the reliability and availability assessment and performance evaluation of complex multistate systems. As an example, we can point to the application to water cooling systems for magnetic resonance inspection [32], multiphase multimotor traction electric drives for an icebreaking ship [33], and many other complex systems [34,35]. Therefore, implementing these methods to explore the performance of FMS, based on reliability features, which consider system flexibility, production setup, aging machines, and multistate problem, is of interest and significant. Consequently, a brief introduction of these two methods is given in the section.

\subsection{Lz-Transform Method}

The Lz-transform method is implemented for the performance evaluation for an FMS aging system in the current article. Lisnianski [36] introduced the method where one can find detailed content and mathematical proofs. A brief introduction comes in the following paragraphs.

A discrete-state continuous-time (DSCT) Markov process $X(t) \in\left\{x_{1}, x_{2}, \ldots, x_{K}\right\}$, which has $K$ possible states and the performance level associated with any state $i$ is $x_{i}, i=1,2, \ldots, K$. The Markov process is completely defined by the set of possible states $\mathbf{x}=\left\{x_{1}, x_{2}, \ldots, x_{K}\right\}$, the transition intensities matrix depend on time $A=\left[a_{i j}\right], i, j=1,2, \ldots, K$ and by the initial states probability distribution given by $\mathbf{p}_{0}=\left\{p_{10}=\operatorname{Pr}\left\{X(0)=x_{1}\right\}, \ldots, p_{K 0}=\operatorname{Pr}\left\{X(0)=x_{K}\right\}\right\}$. Therefore, according to [37], the Lz-transform of a DSCT Markov process $X(t)$ is defined by the following expression:

$$
L_{z}\{X(t)\}=\sum_{i=1}^{K} p_{i}(t) z^{\mathrm{g}_{i}}
$$


where $p_{i}(t)$ is the probability that the process is in state $i$ at time instant $t \geq 0$ for a given initial states probability distribution $\mathbf{p}_{0}, \mathrm{~g}_{i}$ is the performance level of state $i$, and $z$ is a complex variable.

Without losing generality, we consider any element $e$ in a multistate system (MSS) can have $k_{e}$ different states corresponding to different performances, represented by the set $\mathbf{g}_{e}=\left\{\mathrm{g}_{e 1}, \ldots, \mathrm{g}_{e k_{e}}\right\}$, where $\mathrm{g}_{e i}$ is the performance rate of element $e$ in the state $i, i \in\left\{1,2, \ldots, k_{e}\right\}$, and $e \in\{1,2, \ldots, n\}$, where $n$ is the number of elements in the MSS.

The first step of Lz-transform is to build a Markov model of the stochastic process for each multistate element in the MSS. Based on this model, the state probabilities $p_{e i}=\operatorname{Pr}\left\{G_{e}(t)=\mathrm{g}_{e i}\right\}$, $i \in\left\{1,2, \ldots, k_{e}\right\}$ for every MSS's element can be obtained as a solution of the corresponding system of differential equations under the given initial conditions. These probabilities define the output stochastic process $G_{e}(t)$ for each element $e$ in the MSS. Then, the individual Lz-transform for each element $e$ can be found as

$$
L_{z}\left\{G_{e}(t)\right\}=\sum_{i=1}^{k_{e}} p_{e i}(t) z^{\mathrm{g}_{e i}}, e=\{1,2, \ldots, n\} .
$$

Then the next step is based on the previously determined Lz-transform for each element $e$ and system structure function $f$, given by $G(t)=f\left(G_{1}(t), G_{2}(t), \ldots, G_{n}(t)\right)$, where the Lz-transform of the output stochastic process for the entire MSS should be defined. Using the Ushakov's operator $\Omega_{f}$ [38] over all Lz-transforms of individual elements, one can obtain the resulting Lz-transform $L_{z}\{G(t)\}$ associated with output performance stochastic process $G(t)$ of the entire MSS:

$$
L_{z}\{G(t)\}=\Omega_{f}\left\{L_{z}\left[G_{1}(t)\right], L_{z}\left[G_{2}(t)\right], \ldots, L_{z}\left[G_{n}(t)\right]\right\} .
$$

The resulting Lz-transform is associated with output performance stochastic process for the entire MSS:

$$
L_{z}\{G(t)\}=\sum_{k=1}^{K} p_{k}(t) z^{\mathrm{g}_{k}},
$$

and many performance measures of MSS can be easily derived from the resulting Lz-transform. For example, the mean instantaneous performance calculated in the following form:

$$
E(t)=\sum_{\mathrm{g}_{k} \geq 0} p_{k}(t) \mathrm{g}_{k}
$$

\subsection{Markov Reward Approach}

CNC machine tools with various and powerful functions are the main components of FMS and consist of many different assembles. When the failure of CNC machine tools occurs due to one (or a few) assembly(s) or component(s) failing, the subsequent action is minimal repair, which means that the failed system is returned back to a working state that "as bad as old" after the repair [39]. In such a situation, the failure pattern of CNC machine tools can be described by the non-homogeneous Poisson process (NHPP). Based on this, the Markov reward approach that incorporates the time-varying failure rate of aging components is utilized for general reliability measures evaluation. The brief introduction is as follows, and more details and interesting examples can be found in [40].

Howard [41] proposed a Markov reward model in 1960, which was widely used in various theoretical and practical studies [42,43]. The core idea of Markov reward model is as follows: a continuous-time Markov chain with $M$ different states and a transition matrix between states is $\boldsymbol{a}=\left[a_{i j}\right], i, j=1,2, \ldots, M$. It is assumed that if the process stays in any state $i$ during the time unit, a certain amount of reward $r_{i i}$ is achieved. Similarly, it is also assumed that each time the process transits from state $i$ to state $j$ an additional amount of reward $r_{i j}$ is achieved. The rewards may also be negative when it characterizes a loss or penalty. Consequently, for such processes, it is crucial to determine a reward matrix $r=\left[r_{i j}\right], i, j=1,2, \ldots, M$ in additional to a transition intensity matrix. Then, many 
important reliability indexes can be determined by comprehensively applying and calculating these two matrices during the operation of the system.

Let $V_{i}(t)$ be the expected total reward accumulated up to time $t$, given the initial state of the process as time instant $t=0$ is in state $i$. Howard differential equations with time-varying transition intensities $a_{i j}$ should be solved under specified initial conditions to find the total expected rewards.

$$
\frac{d V_{i}(t)}{d t}=r_{i i}+\sum_{\substack{j=1 \\ j \neq i}}^{M} a_{i j} r_{i j}+\sum_{j=1}^{M} a_{i j} V_{j}(t), i, j=1,2, \ldots, M .
$$

In the most common case, the MSS begins to accumulate reward after time instant $t=0$, thus, the initial conditions are

$$
V_{i}(0)=0, i=1,2, \ldots, M \text {. }
$$

For instance, if the state $M$ with the highest performance level is defined as the initial state, the value $V_{M}(t)$ should be found as a solution of system (6).

It was shown in [44] that many reliability measures for aging or non-aging MSS can be found by the determination of rewards in a corresponding reward matrix. Therefore, the construction rules of the reward matrix for each reliability measures are the most critical, which will be demonstrated while calculating relevant indicators.

\section{Multistate Model of the Flexible Manufacturing System}

$\mathrm{CNC}$ machine tools as the fundamental and crucial equipment for most manufacturing systems have been chosen as the element of the workstation of FMS in the current paper. The storage system, such as an intermediate buffer, is not considered in this article since the trend of FMS is to reduce or even cancel the cache area [45]. An FMS is capable of manufacturing various kinds of product families through CNC machine tools, which are loaded and unloaded by robots [46]. Therefore, the first step in the multistate modeling of FMS is to establish a multistate model of CNC machine tools. Moreover, a novel multistate $\mathrm{CNC}$ machine tool model is presented in the current paper.

\subsection{Multistate Model of CNC Machine Tools}

A typical state transition process of CNC machine tools was presented by the authors in [47], which argued that $\mathrm{CNC}$ machine tools were a binary-state system, working and failure state, and the transition between states is caused by faults in different subsystems. This is a binary state transition description of the CNC machine tools that cannot be implemented directly in the current paper because it lacks consideration of the impact of product types on system status. Therefore, to demonstrate the production flexibility of CNC machine tools and the performance influence caused by production setup activities, we assume one product can be processed on machine tool A or B if the corresponding settings are prepared. From the practically technological restrictions, there exists a limitation on time of usage of each task and necessity to change one task to another, so the setup states are required for changing processes. That is, one product capable of being manufactured on two machine tools $\mathrm{A}$ or $\mathrm{B}, E_{A}$ and $E_{B}$ are appointed to $\mathrm{A}$ and $\mathrm{B}$ for the corresponding process, $S_{A B}$ and $S_{B A}$ are built for the changing processes between $A$ and $B$. Note that, $E_{A}$ and $E_{B}$ are binary state systems during the manufacturing period. Thus, the product manufacturing process on CNC machine tools can be extended to a multistate system (MSS) when considering production flexibility. The state transition process of the example in Figure 1 shown as follows: 


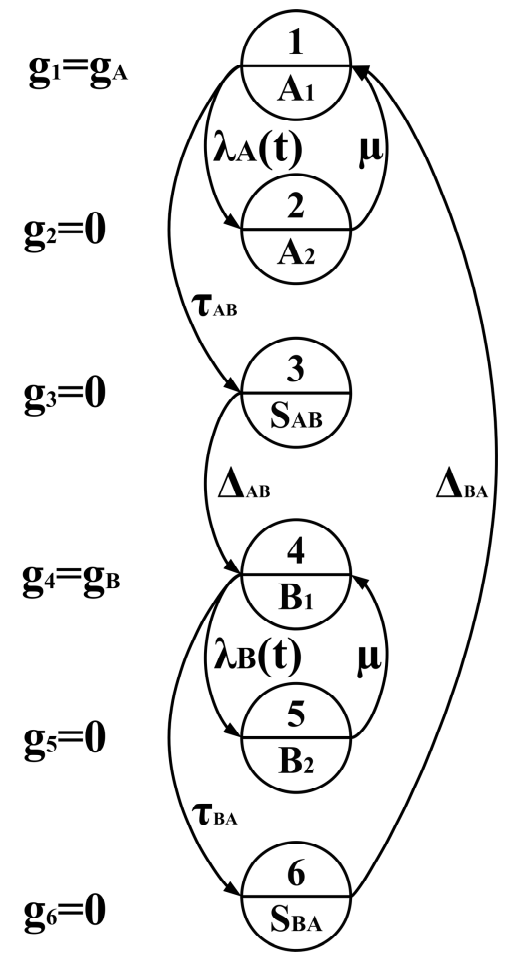

Figure 1. State transition diagram of CNC machine tools A and B.

where,

$i=1, \ldots, 6$ : the number of the state of CNC machine tools,

$\mathrm{g}_{i}, i=1, \ldots, 6$ : the corresponding performance of each state,

$\mathrm{g}_{A}, \mathrm{~g}_{B}$ : the performance of CNC machine tools $\mathrm{A}$ and $\mathrm{B}$ to manufacture, respectively,

$A_{1}, B_{1}$ : working state of machine tools $\mathrm{A}$ and $\mathrm{B}$, respectively,

$A_{2}, B_{2}$ : failure state of machine tools $\mathrm{A}$ and $\mathrm{B}$, respectively,

$S_{A B}$ : the setup state for machine tool B,

$S_{B A}$ : the setup state for machine tool A,

$\lambda_{A}(t), \lambda_{B}(t)$ : failure rates of the machine tools $\mathrm{A}$ and $\mathrm{B}$, which not only indicate the availability of the machine tool is task-based, but the aging characteristic,

$\mu$ : repair rate of the machine tools,

$\tau_{A B}, \tau_{B A}$ : the corresponding transition intensities from working state to setup state, which are random variables and calculated through the total setup times per year,

$\Delta_{A B}, \Delta_{B A}$ : the transition intensities from setup to working state, which is the reverse of the mean time to setup.

The state transition process of CNC machine tools with production flexibility includes two setup states, $S_{A B}$ and $S_{B A}$, which represent one of the states in which the machine tools in the FMS could be in the actual production process. Obviously, only in the $A_{1}$ and $B_{1}$ states is the machine tool in normal working condition (designated as 1), and the rest $A_{2}, B_{2}, S_{A B}, S_{B A}$ are idle (designated as 0 ). Therefore, the state transition intensity matrix $\boldsymbol{a}$ of the $\mathrm{CNC}$ machine tools obtained as follows:

$$
\boldsymbol{a}=\left[\begin{array}{cccccc}
-\left(\lambda_{A}(t)+\tau_{A B}\right) & \lambda_{A}(t) & \tau_{A B} & 0 & 0 & 0 \\
\mu & -\mu & 0 & 0 & 0 & 0 \\
0 & 0 & -\Delta_{A B} & \Delta_{A B} & 0 & 0 \\
0 & 0 & 0 & -\left(\lambda_{B}(t)+\tau_{B A}\right) & \lambda_{B}(t) & \tau_{B A} \\
0 & 0 & 0 & \mu & -\mu & 0 \\
\Delta_{B A} & 0 & 0 & 0 & 0 & -\Delta_{B A}
\end{array}\right] .
$$


Based on the Markov reward approach, to assess the average instantaneous availability $\overline{A_{i}}(t)$ of the CNC machine tools, it is necessary to build the reward matrix $r$, and the corresponding rewards in $r$ can be determined in the following manner:

1. All rewards that indicate acceptable states are defined as 1;

2. The rewards associated with all unacceptable states should be zeroed, as well as the rewards associated with all transitions.

Thus, the reward matrix $r$ of the $\mathrm{CNC}$ machine tools depicted in Figure 1 is

$$
\boldsymbol{r}=\left[\begin{array}{llllll}
1 & 0 & 0 & 0 & 0 & 0 \\
0 & 0 & 0 & 0 & 0 & 0 \\
0 & 0 & 0 & 0 & 0 & 0 \\
0 & 0 & 0 & 1 & 0 & 0 \\
0 & 0 & 0 & 0 & 0 & 0 \\
0 & 0 & 0 & 0 & 0 & 0
\end{array}\right] .
$$

Then, the system of differential equations for cumulative reward calculation can be obtained according to system (6).

$$
\left\{\begin{array}{l}
\frac{d V_{1}(t)}{d t}=1+\lambda_{A}(t) V_{2}(t)+\tau_{A B} V_{3}(t)-\left(\lambda_{A}(t)+\tau_{A B}\right) V_{1}(t) \\
\frac{d V_{2}(t)}{d t}=\mu V_{1}(t)-\mu V_{2}(t) \\
\frac{d V_{3}(t)}{d t}=\Delta_{A B} V_{4}(t)-\Delta_{A B} V_{3}(t) \\
\frac{d V_{4}(t)}{d t}=1+\lambda_{B}(t) V_{5}(t)+\tau_{B A} V_{6}(t)-\left(\lambda_{B}(t)+\tau_{B A}\right) V_{4}(t) \\
\frac{d V_{5}(t)}{d t}=\mu V_{4}(t)-\mu V_{5}(t) \\
\frac{d V_{6}(t)}{d t}=\Delta_{B A} V_{1}(t)-\Delta_{B A} V_{6}(t)
\end{array} .\right.
$$

The mean reward $V_{i}(t)$ accumulated during interval $[0, t]$ defines a time that the CNC machine tools will be in the set of acceptable states in the case where state $i$ is the initial state. This reward should be calculated as a solution of system (8). After solving system (8) and determining $V_{i}(t)$, the CNC machine tools average instantaneous availability $\overline{A_{i}}(t)$ can be found for every $i=1,2, \ldots, M$.

$$
\overline{A_{i}}(t)=V_{i}(t) / t
$$

The usage of the Markov reward approach to assess the average instantaneous availability and failure criticality importance will be presented in Section 4.1.

\subsection{Multistate Model of a Flexible Manufacturing System}

In this section, an analytical model of a flexible manufacturing system, as shown in Figure 2, is discussed. We modeled the system as a discrete state continuous-time Markov process based on the Lz-transform. Then the exact solution of this model was derived to analyze the system performance. 


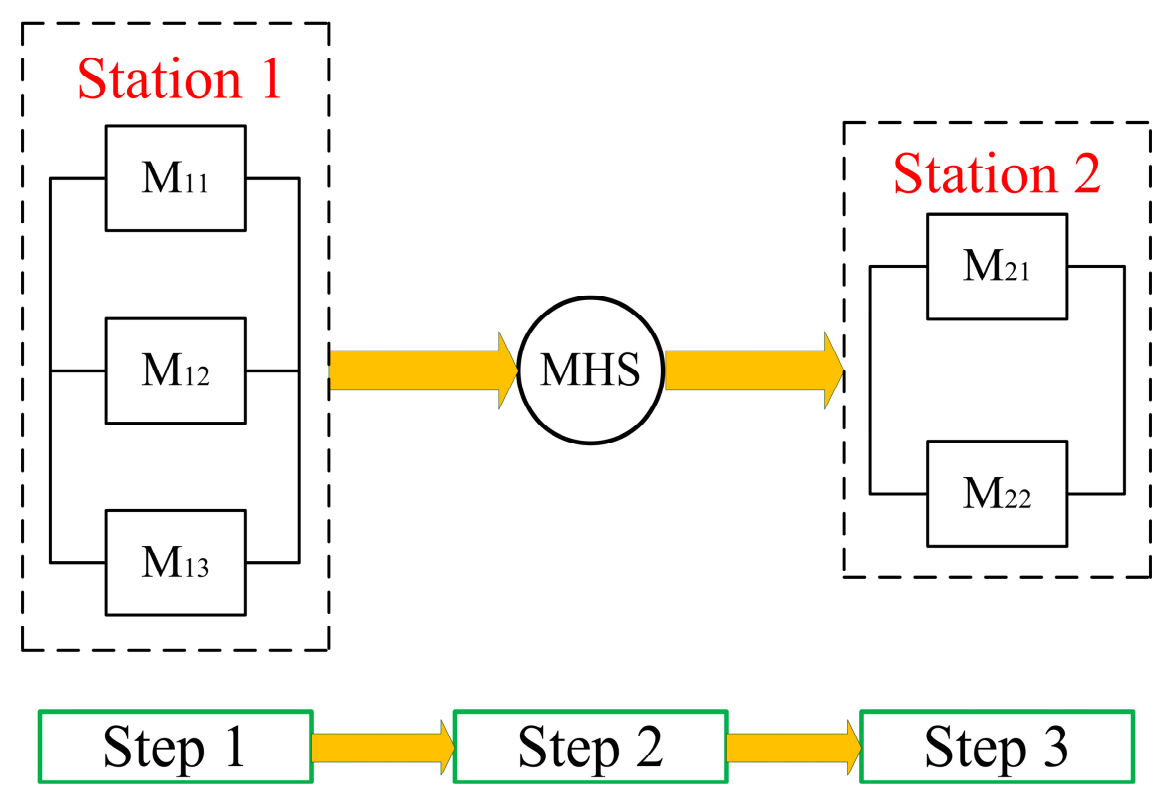

Figure 2. Reliability block diagram of the flexible manufacturing system (FMS) and process to manufacture the product.

\subsubsection{System Description}

The FMS considered in this paper, depicted in Figure 2, consists of three steps to manufacture the required product. Two types of CNC machine tools are used to finish the production task at the first and second stations, which indicates that machines at different stations have different failure mechanisms. The specific running process of FMS is three identical and independent working machines, each consisting of two machine tools $\mathrm{A}$ and $\mathrm{B}$ at the first station to complete the first task of processing. Then the semi-finished products are transported by the MHS, which is considered as a binary-state assembly, to Station 2; finally, two other machines independent CNC machine tools located at Station 2 then process the semi-finished products to obtain the required product. Therefore, the CNC machine tools at each station work essentially in parallel, and each machine tool follows the state transitions process shown in Figure 1. Regarding the whole FMS, which is a serial structure, it is assumed that a synchronization rule exists in the machines at the two stations for completing the products manufacturing process, i.e.,

- Machine synchronization rule: it is required that corresponding machine tools exist for performing A-related operations at two Stations, and the MHS can normally transport the semi-finished products; the same requirement for B-related operations exist.

There are six states for each CNC machine tool and total $6^{3} \times 2 \times 6^{2}=15,552$ states for the FMS if the straightforward Markov method is utilized to evaluate the performance. However, the Lz-transform method can easily overcome this "states explosion problem" and efficiently finish the performance evaluation. 


\subsubsection{Station 1}

Using the state-transitions diagram (Figure 1) in accordance with the Markov method, and the parameters designated as $\lambda_{A}^{S 1}(t), \lambda_{B}^{S 1}(t), \tau_{A B^{\prime}}^{S 1} \tau_{B A^{\prime}}^{S 1} \mu^{S 1}, \Delta_{A B^{\prime}}^{S 1} \Delta_{B A^{\prime}}^{S 1}$, we build the following system of differential equations for CNC machine tool $m, m=1,2,3$ at Station 1:

$$
\left\{\begin{array}{l}
\frac{d p_{m 1}^{S 1}(t)}{d t}=-\left(\lambda_{A}^{S 1}(t)+\tau_{A B}^{S 1}\right) p_{m 1}^{S 1}(t)+\mu^{S 1} p_{m 2}^{S 1}(t)+\Delta_{B A}^{S 1} p_{m 6}^{S 1}(t) \\
\frac{d p_{m 2}^{S 1}(t)}{d t}=\lambda_{A}^{S 1}(t) p_{m 1}^{S 1}(t)-\mu^{S 1} p_{m 2}^{S 1}(t) \\
\frac{d p_{m 3}^{S 1}(t)}{d t}=\tau_{A B}^{S 1} p_{m 1}^{S 1}(t)-\Delta_{A B}^{S 1} p_{m 3}^{S 1}(t) \\
\frac{d p_{m 4}^{S 1}(t)}{d t}=-\left(\lambda_{B}^{S 1}(t)+\tau_{B A}^{S 1}\right) p_{m 4}^{S 1}(t)+\Delta_{A B}^{S 1} p_{m 3}^{S 1}(t)+\mu^{S 1} p_{m 5}^{S 1}(t) \\
\frac{d p_{m 5}^{S 1}(t)}{d t}=\lambda_{B}^{S 1}(t) p_{m 4}^{S 1}(t)-\mu^{S 1} p_{m 5}^{S 1}(t) \\
\frac{d p_{m 6}^{S 1}(t)}{d t}=\tau_{B A}^{S 1} p_{m 4}^{S 1}(t)-\Delta_{B A}^{S 1} p_{m 6}^{S 1}(t)
\end{array}\right.
$$

Initial conditions are $p_{m 1}^{S 1}(0)=1, p_{m 2}^{S 1}(0)=p_{m 3}^{S 1}(0)=p_{m 4}^{S 1}(0)=p_{m 5}^{S 1}(0)=p_{m 5}^{S 1}(0)=0$; or $p_{m 4}^{S 1}(0)=1, p_{m 1}^{S 1}(0)=p_{m 2}^{S 1}(0)=p_{m 3}^{S 1}(0)=p_{m 5}^{S 1}(0)=p_{m 5}^{S 1}(0)=0$. The reason for the two initial conditions is that it is reasonable to start producing any kind of product at the beginning of the production process. The difference between the two initial conditions can be analyzed by productivity comparison.

A numerical solution for probabilities $p_{m i}^{S 1}(t), m=1,2,3, i=1,2,3,4,5,6$ can be calculated for this system of differential equations using MATLAB ${ }^{\circledR}$. Therefore, for one CNC machine tool at Station 1, we can obtain the following output performance stochastic process:

$$
\left\{\begin{array}{c}
\mathbf{g}_{m}^{S 1}=\left\{\mathrm{g}_{m 1}^{S 1}, \mathrm{~g}_{m 2}^{S 1}, \mathrm{~g}_{m 3^{\prime}}^{S 1} \mathrm{~g}_{m 4^{\prime}}^{S 1} \mathrm{~g}_{m 5}^{S 1}, \mathrm{~g}_{m 6}^{S 1}\right\} \\
\mathbf{p}_{m}^{S 1}(t)=\left\{p_{m 1}^{S 1}(t), p_{m 2}^{S 1}(t), p_{m 3}^{S 1}(t), p_{m 4}^{S 1}(t), p_{m 5}^{S 1}(t), p_{m 6}^{S 1}(t)\right\}
\end{array}, m=1,2,3 .\right.
$$

Having the sets $\mathbf{g}_{m}^{S 1}, \mathbf{p}_{m}^{S 1}(t), m=1,2,3$, one can define for a CNC machine tool the Lz-transform associated with the machine output performance stochastic process.

$$
\begin{aligned}
L_{z}\left\{\mathbf{g}_{m}^{S 1}(t)\right\}=p_{m 1}^{S 1}(t) z^{\mathrm{g}_{m 1}^{S 1}}+ & p_{m 2}^{S 1}(t) z^{\mathrm{g}_{m 2}^{S 1}}+p_{m 3}^{S 1}(t) z^{\mathrm{g}_{m 3}^{S 1}}+p_{m 4}^{S 1}(t) z^{\mathrm{g}_{m 4} 11}+p_{m 5}^{S 1}(t) z^{\mathrm{g}_{m 5}}+ \\
& p_{m 6}^{S 1}(t) z^{\mathrm{g}_{m 6}^{S 1}}, m=1,2,3 .
\end{aligned}
$$

As CNC machine tools work in parallel at Station 1, the Lz-transform associated with Station 1 is

$$
L_{z}\left\{G^{S 1}(t)\right\}=\Omega_{\text {fpar }}\left(L_{z}\left\{\mathbf{g}_{1}^{S 1}(t)\right\}, L_{z}\left\{\mathbf{g}_{2}^{S 1}(t)\right\}, L_{z}\left\{\mathbf{g}_{3}^{S 1}(t)\right\}\right) .
$$

\subsubsection{Material Handling System (MHS)}

The devices work in the MHS are regarded as a whole system, for which the state-transitions diagram is shown in Figure 3, where 1 indicates the working state of the device while 0 means failure state. Then using Figure 3 in accordance with the Markov method, we build the following system of differential equations for MHS:

$$
\left\{\begin{array}{l}
\frac{d p_{1}^{M H S}(t)}{d t}=-\lambda_{M H S} p_{1}^{M H S}(t)+\mu_{M H S} p_{2}^{M H S}(t) \\
\frac{d p_{2}^{M H S}(t)}{d t}=\lambda_{M H S} p_{1}^{M H S}(t)-\mu_{M H S} p_{2}^{M H S}(t)
\end{array},\right.
$$


where $\lambda_{M H S}$ is the failure rate of the MHS and $\mu_{M H S}$ is the corresponding repair rate. Initial conditions are $p_{1}^{M H S}(0)=1, p_{2}^{M H S}(0)=0$.

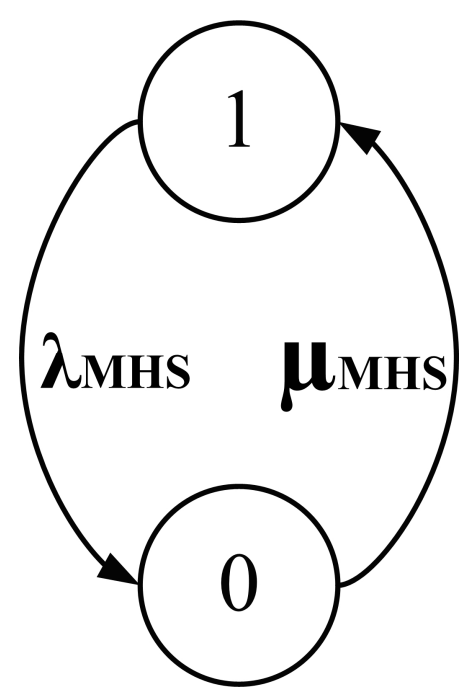

Figure 3. State transition diagram of the material handling system (MHS).

A numerical solution for probabilities $p_{i}^{M H S}(t), i=1,2$ can be calculated for this system of differential equations using MATLAB ${ }^{\circledR}$. Therefore, for the MHS, we can obtain the following output performance stochastic process:

$$
\left\{\begin{array}{c}
\mathbf{g}^{M H S}=\left\{\mathrm{g}_{1}^{M H S}, \mathrm{~g}_{2}^{M H S}\right\} \\
\mathbf{p}^{M H S}(t)=\left\{p_{1}^{M H S}(t), p_{2}^{M H S}(t)\right\}
\end{array} .\right.
$$

Having the sets $\mathbf{g}^{M H S}, \mathbf{p}^{M H S}(t)$, one can define the Lz-transform, associated with the MHS output performance stochastic process.

$$
L_{z}\left\{G^{M H S}(t)\right\}=p_{1}^{M H S}(t) z^{\mathrm{g}^{M H S}}+p_{2}^{M H S}(t) z^{\mathrm{g}_{2}^{M H S}} .
$$

\subsubsection{Station 2}

Obviously, the Lz-transform associated with Station 2 could be constructed in a similar way to Station 1. Note that, the difference in the construction of the Lz-transform associated with Station 2 is that the parameters of CNC machine tools at Station 2 are different from that at Station 1. Here, the parameters defined as $\lambda_{A}^{S 2}(t), \lambda_{B}^{S 2}(t), \tau_{A B^{\prime}}^{S 2} \tau_{B A^{\prime}}^{S 2} \mu^{S 2}, \Delta_{A B}^{S 2}, \Delta_{B A}^{S 2}$. Therefore, for one CNC machine tool at Station 2 we can obtain the following output performance stochastic process by the similar method using at Station 1:

$$
\left\{\begin{array}{c}
\mathbf{g}_{m}^{S 2}=\left\{\mathrm{g}_{m 1}^{S 2}, \mathrm{~g}_{m 2^{\prime}}^{S 2} \mathrm{~g}_{m 3^{\prime}}^{S 2} \mathrm{~g}_{m 4^{\prime}}^{S 2} \mathrm{~g}_{m 5^{\prime}}^{S 2} \mathrm{~g}_{m 6}^{S 2}\right\} \\
\mathbf{p}_{m}^{S 2}(t)=\left\{p_{m 1}^{S 2}(t), p_{m 2}^{S 2}(t), p_{m 3}^{S 2}(t), p_{m 4}^{S 2}(t), p_{m 5}^{S 2}(t), p_{m 6}^{S 2}(t)\right\}
\end{array}, m=1,2 .\right.
$$

Having the sets $\mathbf{g}_{m}^{S 2}, \mathbf{p}_{m}^{S 2}(t), m=1,2$ one can define for a CNC machine tool Lz-transform associated with the machine output performance stochastic process.

$$
L_{z}\left\{\mathrm{~g}_{m}^{S 2}(t)\right\}=p_{m 1}^{S 2}(t) z^{\mathrm{g}_{m 1}^{S 2}}+p_{m 2}^{S 2}(t) z^{\mathrm{g}_{m 2}^{S 2}}+p_{m 3}^{S 2}(t) z^{\mathrm{g}_{m 3}^{S 2}}+p_{m 4}^{S 2}(t) z^{\mathrm{g}_{m 4} 2}+p_{m 5}^{S 2}(t) z^{\mathrm{g}_{m 5}^{S 2}}+p_{m 6}^{S 2}(t) z^{\mathrm{g}_{m 6}} .
$$


As CNC machine tools are work in parallel at Station 2, the Lz-transform associated with the Station 2 is

$$
L_{z}\left\{G^{S 2}(t)\right\}=\Omega_{\text {fpar }}\left(L_{z}\left\{\mathbf{g}_{1}^{S 2}(t)\right\}, L_{z}\left\{\mathbf{g}_{2}^{S 2}(t)\right\}\right) .
$$

\section{Illustrated Case}

To demonstrate details about the efficiency and functionality of the proposed method and model in analyzing of the performance of flexible manufacturing systems, a case is illustrated in this section based on the FMS that is depicted in the above section. The parameters for calculating are shown in Table 1.

Table 1. The related parameters for equipment at each station.

\begin{tabular}{cccccccccccc}
\hline & $\lambda_{A}$ & $\lambda_{B}$ & $\lambda_{M H S}$ & $\mu$ & $\tau_{A B}$ & $\tau_{B A}$ & $\Delta_{A B}$ & $\Delta_{B A}$ & $\mathbf{g}_{A}$ & $\mathbf{g}_{B}$ & $\mathbf{g}_{M H S}$ \\
\hline Station 1 & $\lambda_{A}^{S 1}(t)=\frac{2.2}{1200}\left(\frac{t}{1200}\right)^{1.2}$ & $\lambda_{B}^{S 1}(t)=\frac{1.8}{1000}\left(\frac{t}{1000}\right)^{0.8}$ & & $\frac{1}{4}$ & $\frac{2}{500}$ & $\frac{3}{500}$ & $\frac{1}{5}$ & $\frac{1}{6}$ & 6 & 5 \\
\hline MHS & & & $\lambda_{M H S}=0.0002$ & $\frac{1}{2}$ & & & & & 20 \\
\hline Station 2 & $\lambda_{A}^{S 2}(t)=\frac{2.7}{1500}\left(\frac{t}{1500}\right)^{1.7}$ & $\lambda_{B}^{S 2}(t)=\frac{2.1}{1300}\left(\frac{t}{1300}\right)^{1.1}$ & & $\frac{1}{3}$ & $\frac{2}{500}$ & $\frac{3}{500}$ & $\frac{1}{3}$ & $\frac{1}{4}$ & 12 & 10 & \\
\hline
\end{tabular}

Note, the total operating time of the FMS is $5000 \mathrm{~h}$ per year, and the materials for production are saturated. The failure process of CNC machine tools follows the Weibull distribution, which has been widely utilized in the reliability evaluation of that system. The failure of the MHS occurs randomly and obeys the exponential distribution; there is no relation with the product type as well. The repair rates of all machines are set as constants, which are the reciprocal of the mean time to repair, respectively. The meaning and calculation of $\tau_{A B}, \tau_{B A}$ and $\Delta_{A B}, \Delta_{B A}$ are as described in Section 3.1. However, note that the machine tools at both stations need to be changed simultaneously when the setup preparation is required for any product. Thus, $\tau_{A B}$ and $\tau_{B A}$ take the same value for the machines at two stations. Finally, $g_{A}$ and $g_{B}$ represent the performance level of each machine, working normally for one hour. In accordance with the setting parameters, one can perform performance analysis on the CNC machine tools and FMS, including the setup state.

\subsection{Performance Analysis of Multistate CNC Machine Tools}

Taking a CNC machine tool at Station 1 as an example, the performance of this system can be evaluated through the Markov reward approach. According to the cumulative reward differential Equation (8) and the formula for CNC machine tools' average instantaneous availability (9), the availability calculation of the machine is presented in Figure 4.

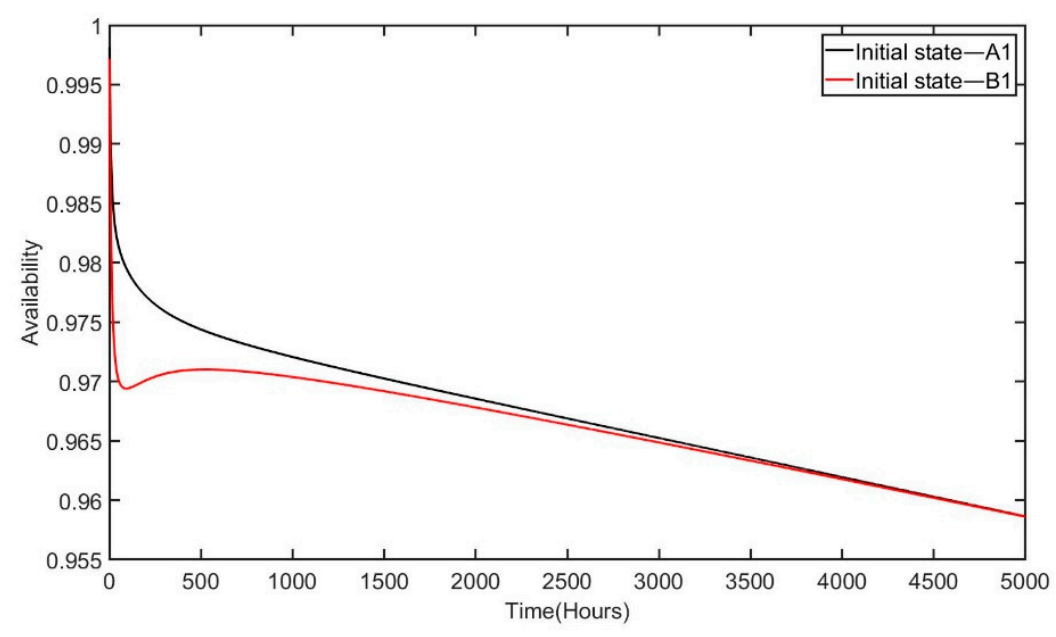

Figure 4. The average instantaneous availability of one CNC machine tool under different initial states. 
In Figure 4, system availability performed better when the state $A_{1}$ was the initial state than that with $B_{1}$ in the early phase of the year, which can be used as a basis for developing a production plan to avoid the additional downtime losses.

Afterward, it is significant to compare the contribution of each failure state (the setup state also regarded as a failure) to the system downtime, which helps optimize the production cost. Therefore, the failure criticality importance (FCI) $[48,49]$ is introduced to calculate the percentage of each failure state to the system downtime. The FCI can be obtained as follows:

$$
I_{F C I}(j ; t)=\frac{\left(\begin{array}{c}
\text { number of system failures, } \\
\text { caused by subsystem } j \text { in }[0, t]
\end{array}\right)}{(\text { number of system failures in }[0, t])}
$$

where $I_{F C I}(j ; t)$ is the failure criticality importance of the $j$ th failure state in $[0, t]$. Subsequently, the key is to evaluate the number of system failures and the number of the system entrance of each down state, which can be obtained through the Markov reward approach. In this case, the mean accumulated reward $V_{i}(t)$ obtained by solving (6) provides the mean number of entrances into the failure state in the time interval $[0, t]$. The reward matrix formulated under the following rules:

1. All rewards that indicate an acceptable state transfer to an unacceptable state are defined as 1;

2. The other awards in the matrix are defined as 0 .

Then, the reward matrixes for the number of the system entrance of each down state are:

$$
\begin{aligned}
& r_{F C I}^{A_{2}}=\left[\begin{array}{llllll}
0 & 1 & 0 & 0 & 0 & 0 \\
0 & 0 & 0 & 0 & 0 & 0 \\
0 & 0 & 0 & 0 & 0 & 0 \\
0 & 0 & 0 & 0 & 0 & 0 \\
0 & 0 & 0 & 0 & 0 & 0 \\
0 & 0 & 0 & 0 & 0 & 0
\end{array}\right], r_{F C I}^{S_{A B}}=\left[\begin{array}{llllll}
0 & 0 & 1 & 0 & 0 & 0 \\
0 & 0 & 0 & 0 & 0 & 0 \\
0 & 0 & 0 & 0 & 0 & 0 \\
0 & 0 & 0 & 0 & 0 & 0 \\
0 & 0 & 0 & 0 & 0 & 0 \\
0 & 0 & 0 & 0 & 0 & 0
\end{array}\right], \\
& r_{F C I}^{B_{2}}=\left[\begin{array}{llllll}
0 & 0 & 0 & 0 & 0 & 0 \\
0 & 0 & 0 & 0 & 0 & 0 \\
0 & 0 & 0 & 0 & 0 & 0 \\
0 & 0 & 0 & 0 & 1 & 0 \\
0 & 0 & 0 & 0 & 0 & 0 \\
0 & 0 & 0 & 0 & 0 & 0
\end{array}\right], r_{F C I}^{S_{A B}}=\left[\begin{array}{llllll}
0 & 0 & 0 & 0 & 0 & 0 \\
0 & 0 & 0 & 0 & 0 & 0 \\
0 & 0 & 0 & 0 & 0 & 0 \\
0 & 0 & 0 & 0 & 0 & 1 \\
0 & 0 & 0 & 0 & 0 & 0 \\
0 & 0 & 0 & 0 & 0 & 0
\end{array}\right] .
\end{aligned}
$$

Therefore, the reward should be found first as a solution of system (6) based on these matrixes and the system state transition intensity matrix $\boldsymbol{a}$. The FCI of each failure state can then be calculated by Equation (21); the results shown in Figure 5: 


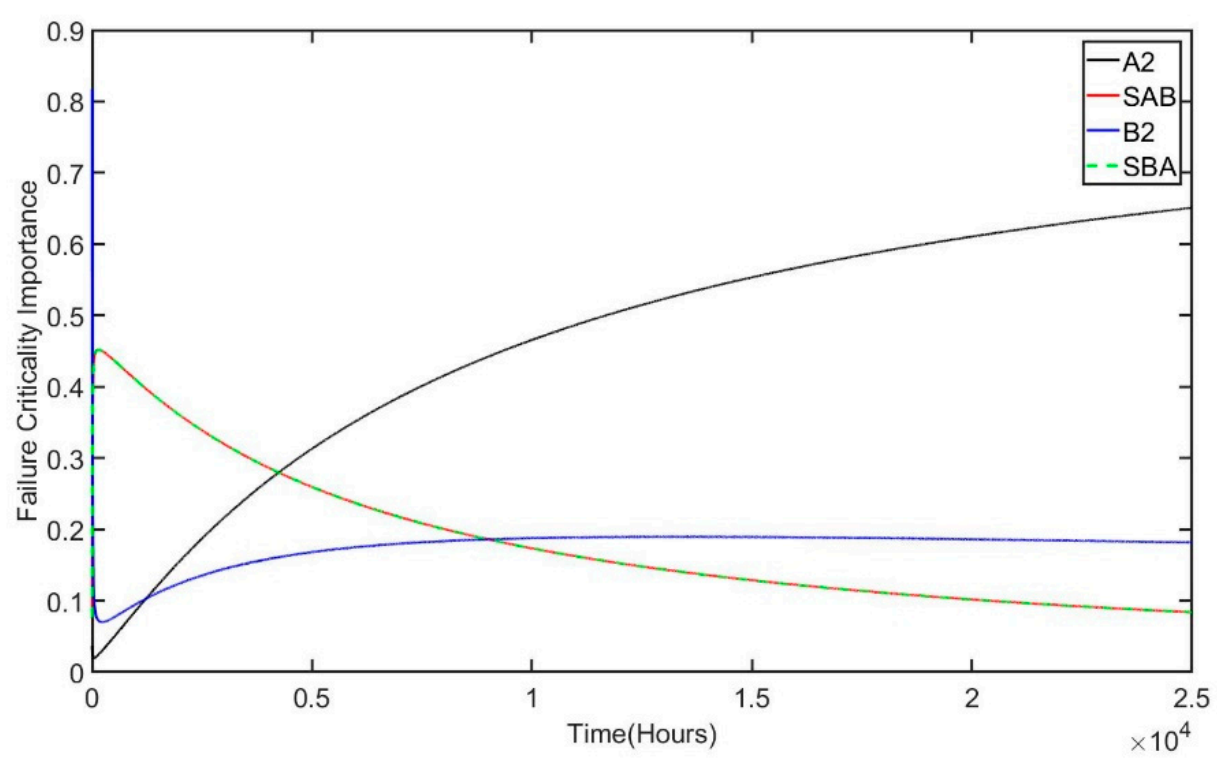

Figure 5. Failure criticality importance of each non-working state.

For the CNC machine tools at Station 1, the main cause of early system downtime was the setup states, and the effects of the two-setup states were always consistent, but the impacts continued to decrease as the system operated longer. Simultaneously, the contributions from the system's failures kept increasing, which is in line with the aging characteristics of the machines.

\subsection{Performance Analysis of Multistate FMS}

In this subsection, the performance of FMS can be obtained by using the Lz-transform method in which the states' probabilities of CNC machine tools at Station 1,2 and that of devices in the MHS are calculated by system (10) and (14) under the designed initial state, respectively.

Thus, in accordance with Equation (12) and parameters shown in Table 1, the Lz-transform for all three identical CNC machine tools at Station 1 have the same rewritten form (21). From here for simplification we substitute $p_{m i}$ with $p_{i}$.

$$
L_{z}\left\{\mathbf{g}_{m}^{S 1}(t)\right\}=p_{1}^{S 1}(t) z^{6}+p_{4}^{S 1}(t) z^{5}+\left(p_{2}^{S 1}(t)+p_{3}^{S 1}(t)+p_{5}^{S 1}(t)+p_{6}^{S 1}(t)\right) z^{0} .
$$

We will use $\Lambda^{S 1}$ to denote $\left(p_{2}^{S 1}(t)+p_{3}^{S 1}(t)+p_{5}^{S 1}(t)+p_{6}^{S 1}(t)\right)$. Consequently, the mean instantaneous performance of one CNC machine tool at Station 1 can be easily derived from the modified Lz-transform in the following form:

$$
E(t)=\sum_{\mathrm{g}_{i}^{S 1} \geq 0} p_{i}^{S 1}(t) \mathrm{g}_{i}^{S 1}, i=1, \ldots, 6 .
$$

Output performance that is manufactured by the $\mathrm{CNC}$ machine tools at Station 1 is depicted in Figure 6. 


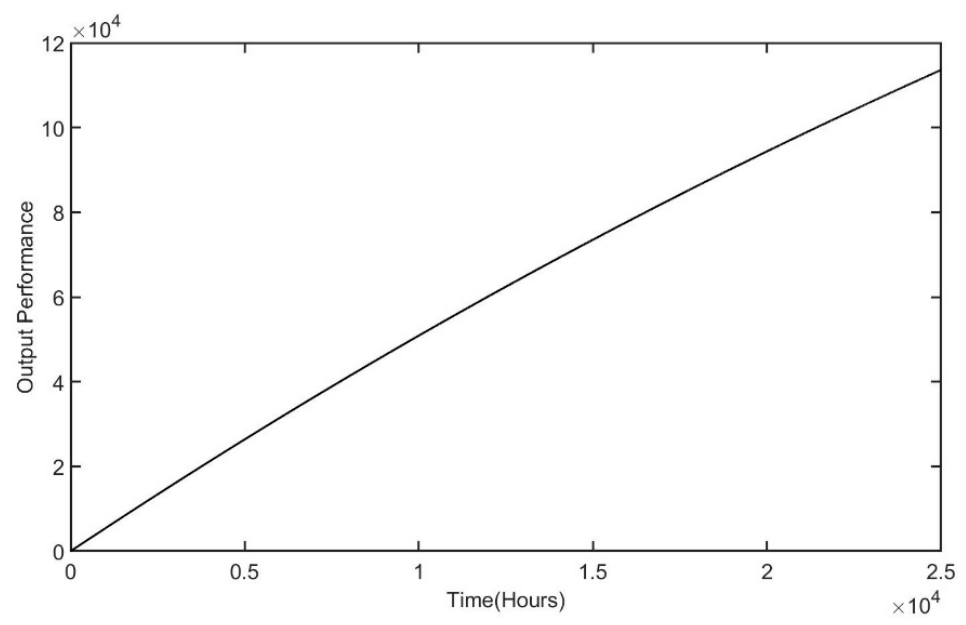

Figure 6. Output of product manufactured by one machine at Station 1.

Using Equation (13) and the rewritten Lz-transform for one CNC machine tool at Station 1, one can obtain the Lz-transform, associated with the whole of Station 1 as

$$
\begin{aligned}
L_{z}\left\{G^{S 1}(t)\right\}=\Omega_{\text {fpar }} & \left(L_{z}\left\{\mathbf{g}_{1}^{S 1}(t)\right\}, L_{z}\left\{\mathbf{g}_{2}^{S 1}(t)\right\}, L_{z}\left\{\mathbf{g}_{3}^{S 1}(t)\right\}\right) \\
& =P_{1}^{S 1}(t) z^{18}+P_{2}^{S 1}(t) z^{17}+P_{3}^{S 1}(t) z^{16}+P_{4}^{S 1}(t) z^{15}+P_{5}^{S 1}(t) z^{12} \\
& +P_{6}^{S 1}(t) z^{11}+P_{7}^{S 1}(t) z^{10}+P_{8}^{S 1}(t) z^{6}+P_{9}^{S 1}(t) z^{5}+P_{10}^{S 1}(t) z^{0} .
\end{aligned}
$$

The meaning of notations are

$$
\begin{aligned}
& P_{1}^{S 1}(t)=p_{1}^{S 1}(t) p_{1}^{S 1}(t) p_{1}^{S 1}(t) \\
& P_{2}^{S 1}(t)=3 p_{1}^{S 1}(t) p_{1}^{S 1}(t) p_{4}^{S 1}(t) \\
& P_{3}^{S 1}(t)=3 p_{1}^{S 1}(t) p_{4}^{S 1}(t) p_{4}^{S 1}(t) \\
& P_{4}^{S 1}(t)=p_{4}^{S 1}(t) p_{4}^{S 1}(t) p_{4}^{S 1}(t) \\
& P_{5}^{S 1}(t)=3 p_{1}^{S 1}(t) p_{1}^{S 1}(t) \Lambda^{S 1} \\
& P_{6}^{S 1}(t)=6 p_{1}^{S 1}(t) p_{4}^{S 1}(t) \Lambda^{S 1} \\
& P_{7}^{S 1}(t)=3 p_{4}^{S 1}(t) p_{4}^{S 1}(t) \Lambda^{S 1} \\
& P_{8}^{S 1}(t)=3 p_{1}^{S 1}(t) \Lambda^{S 1} \Lambda^{S 1} \\
& P_{9}^{S 1}(t)=3 p_{4}^{S 1}(t) \Lambda^{S 1} \Lambda^{S 1} \\
& P_{10}^{S 1}(t)=\Lambda^{S 1} \Lambda^{S 1} \Lambda^{S 1}
\end{aligned}
$$

Similarly, the Lz-transform for the two identical CNC machine tools at Station 2 have the same rewritten form as

$$
L_{z}\left\{\boldsymbol{g}_{q}^{S 2}(t)\right\}=p_{1}^{S 2}(t) z^{12}+p_{4}^{S 2}(t) z^{10}+\Lambda^{S 2} z^{0}, q=1,2,
$$

where $\Lambda^{S 2}$ represents the $\left(p_{2}^{S 2}(t)+p_{3}^{S 2}(t)+p_{5}^{S 2}(t)+p_{6}^{S 2}(t)\right)$. Therefore, the Lz-transform associated with the whole of Station 2 can be derived from Equation (19) as

$$
\begin{aligned}
L_{z}\left\{G^{S 2}(t)\right\}=\Omega_{\text {fpar }} & \left(L_{z}\left\{\mathbf{g}_{1}^{S 2}(t)\right\}, L_{z}\left\{\mathbf{g}_{2}^{S 2}(t)\right\}\right) \\
& =P_{1}^{S 2}(t) z^{24}+P_{2}^{S 2}(t) z^{22}+P_{3}^{S 2}(t) z^{20}+P_{4}^{S 2}(t) z^{12}+P_{5}^{S 2}(t) z^{10} \\
& +P_{6}^{S 2}(t) z^{0} .
\end{aligned}
$$


The notations represent:

$$
\begin{aligned}
& P_{1}^{S 2}(t)=p_{1}^{S 2}(t) p_{1}^{S 2}(t) ; \\
& P_{2}^{S 2}(t)=2 p_{1}^{S 2}(t) p_{4}^{S 2}(t) ; \\
& P_{3}^{S 2}(t)=p_{4}^{S 2}(t) p_{4}^{S 2}(t) ; \\
& P_{4}^{S 2}(t)=2 p_{1}^{S 2}(t) \Lambda^{S 2} ; \\
& P_{5}^{S 2}(t)=2 p_{4}^{S 2}(t) \Lambda^{S 2} ; \\
& P_{6}^{S 2}(t)=\Lambda^{S 2} \Lambda^{S 2}
\end{aligned}
$$

Based on Equation (19), the Lz-transform associated with the whole system can be obtained as

$$
\begin{aligned}
L_{z}\left\{G_{F M S}(t)\right\}= & \Omega_{f s e r}\left(L_{z}\left\{G^{S 1}(t)\right\}, L_{z}\left\{G^{M H S}(t)\right\}, L_{z}\left\{G^{S 2}(t)\right\}\right) \\
& =\Omega_{f s e r}\left(P_{1}^{S 1}(t) z^{18}+P_{2}^{S 1}(t) z^{17}+P_{3}^{S 1}(t) z^{16}+P_{4}^{S 1}(t) z^{15}+P_{5}^{S 1}(t) z^{12}\right. \\
& +P_{6}^{S 1}(t) z^{11}+P_{7}^{S 1}(t) z^{10}+P_{8}^{S 1}(t) z^{6}+P_{9}^{S 1}(t) z^{5} \\
& +P_{10}^{S 1}(t) z^{0}, p_{1}^{M H S}(t) z^{20}+p_{2}^{M H S}(t) z^{0}, P_{1}^{S 2}(t) z^{24}+P_{2}^{S 2}(t) z^{22} \\
& \left.+P_{3}^{S 2}(t) z^{20}+P_{4}^{S 2}(t) z^{12}+P_{5}^{S 2}(t) z^{10}+P_{6}^{S 2}(t) z^{0}\right) .
\end{aligned}
$$

Due to the FMS possessing the characteristic of providing multiple products or services, it is necessary to carry out the performance analysis based on product or service type. Thus, based on the synchronization rule, Equation (26), and simple algebra, where the powers of $z$ are found as minimum values of the powers of the corresponding terms, the Lz-transform associated with the FMS can be obtained as

$$
\begin{aligned}
L_{z}\left\{G_{F M S}(t)\right\}= & P_{1}^{F M S}(t) z^{18}+P_{2}^{F M S}(t) z^{17}+P_{3}^{F M S}(t) z^{16}+P_{4}^{F M S}(t) z^{15}+P_{5}^{F M S}(t) z^{12} \\
& +P_{6}^{F M S}(t) z^{11}+P_{7}^{F M S}(t) z^{10}+P_{8}^{F M S}(t) z^{6}+P_{9}^{F M S}(t) z^{5}+P_{10}^{F M S}(t) z^{0},
\end{aligned}
$$

where:

$$
\begin{aligned}
& P_{1}^{F M S}(t)=P_{1}^{S 1}(t) p_{1}^{M H S}(t) P_{1}^{S 2}(t) ; \\
& P_{2}^{F M S}(t)=P_{2}^{S 1}(t) p_{1}^{M H S}(t) P_{2}^{S 2}(t) ; \\
& P_{3}^{F M S}(t)=P_{3}^{S 1}(t) p_{1}^{M H S}(t) P_{3}^{S 2}(t) ; \\
& P_{4}^{F M S}(t)=P_{4}^{S 1}(t) p_{1}^{M H S}(t) P_{5}^{S 2}(t) ; \\
& P_{5}^{F M S}(t)=P_{5}^{S 1}(t) p_{1}^{M H S}(t)\left(P_{1}^{S 2}(t)+P_{2}^{S 2}(t)+P_{4}^{S 2}(t)\right) ; \\
& P_{6}^{F M S}(t)=P_{6}^{S 1}(t) p_{1}^{M H S}(t) P_{2}^{S 2}(t) ; \\
& P_{7}^{F M S}(t)=P_{7}^{S 1}(t) p_{1}^{M H S}(t)\left(P_{2}^{S 2}(t)+P_{3}^{S 2}(t)+P_{5}^{S 2}(t)\right) ; \\
& P_{8}^{F M S}(t)=P_{8}^{S 1}(t) p_{1}^{M H S}(t)\left(P_{1}^{S 2}(t)+P_{2}^{S 2}(t)+P_{4}^{S 2}(t)\right) ; \\
& P_{9}^{F M S}(t)=P_{9}^{S 1}(t) p_{1}^{M H S}(t)\left(P_{2}^{S 2}(t)+P_{3}^{S 2}(t)+P_{5}^{S 2}(t)\right) ; \\
& P_{10}^{F M S}(t)=1-\sum_{i=1}^{9} P_{i}^{F M S}(t) .
\end{aligned}
$$


The mean instantaneous output performance of product per unit time manufactured by the FMS can be derived according to Equation (5) as

$$
\begin{aligned}
E(t)=18 P_{1}^{F M S}(t) & +15 P_{4}^{F M S}(t)+12\left(P_{2}^{F M S}(t)+P_{5}^{F M S}(t)\right)+10\left(P_{3}^{F M S}(t)+P_{7}^{F M S}(t)\right) \\
& +6\left(P_{3}^{F M S}(t)+P_{6}^{F M S}(t)+P_{8}^{F M S}(t)\right) \\
& +5\left(P_{2}^{F M S}(t)+P_{6}^{F M S}(t)+P_{9}^{F M S}(t)\right) .
\end{aligned}
$$

The productivity calculations of product made by the FMS are presented in Figure 7.

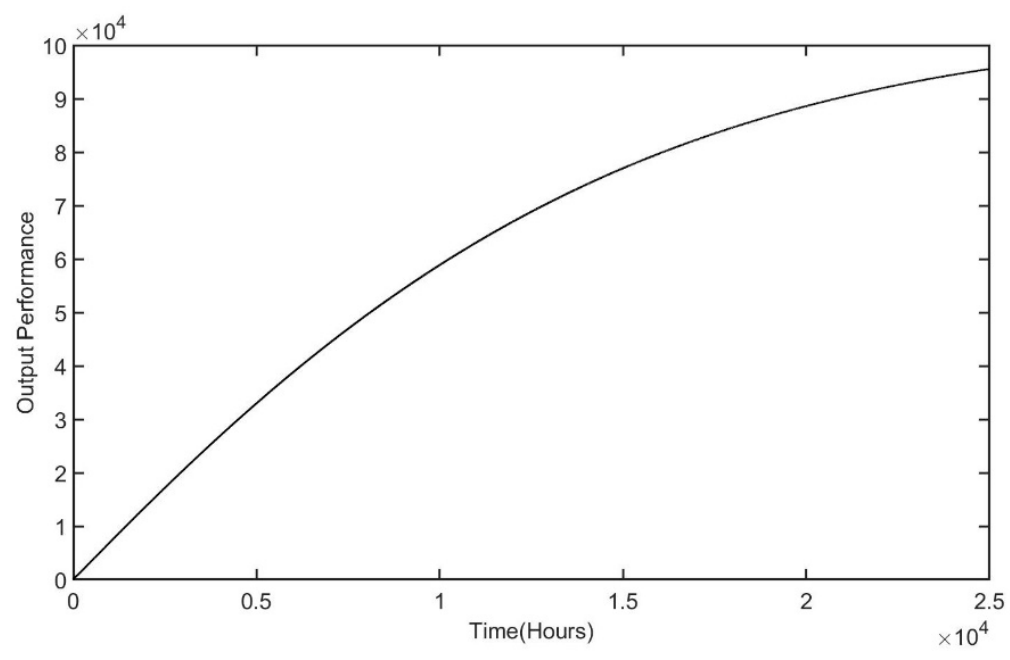

Figure 7. Productivities of product manufactured by the whole FMS.

The evaluation process and results indicate that the random setup commands had a significant impact on the performance of the FMS. How to predict and design optimal production plans based on the proposed methods for satisfying the demand will be an interesting research topic.

\subsection{Availability Analysis of the FMS}

The availability of an FMS can be defined as the ability of the manufacturing system to meet production demands in accordance with specified conditions and time interval [16]. Therefore, the availability of FMS depends on the relation between the output performance and the desired level of this performance-demand, which is determined outside the system. In a general case, demand $W(t)$ is also a random process that can take discrete values from the set $w=\left\{w_{1}, \ldots, w_{M}\right\}$. The desired relation between the system performance and the demand at any time instant $t$ can be expressed by the acceptability function $\Phi(G(t), W(t))$. The acceptable system states correspond to $\Phi(G(t), W(t)) \geq 0$ and the unacceptable states correspond to $\Phi(G(t), W(t))<0$. The last inequality defines the MSS failure criterion. In many practical cases, the MSS performance should be equal to or exceed the demand. Therefore, in such cases, the acceptability function takes the following form:

$$
\Phi(G(t), W(t))=G(t)-W(t),
$$

and the criterion of state acceptability can be expressed as

$$
\Phi(G(t), W(t))=G(t)-W(t) \geq 0 .
$$

In the FMS, without loss of generality, we assume that the required demand level is constant $W(t) \equiv w$ and all system states with performance greater than or equal to $w$ corresponds to the set 
of acceptable states, and all system states with performance lower than $w$ correspond to the set of unacceptable states.

Then combined with Equation (4), the instantaneous availability $A(t)$ of the FMS is the probability that the FMS at instant $t>0$ is in one of the acceptable states.

$$
A(t)=\sum_{\mathrm{g}_{k} \geq w} p_{k}(t)
$$

Consequently, the system of equations of the instantaneous availability of the FMS in different demand is expressed as follows:

$$
\left\{\begin{array}{l}
A_{1}(t)=P_{1}^{F M S}(t), w_{1}=18 \\
A_{2}(t)=\sum_{i=1}^{3} P_{i}^{F M S}(t), w_{2}=16 \\
A_{3}(t)=\sum_{i=1}^{4} P_{i}^{F M S}(t), w_{3}=12 \\
A_{4}(t)=\sum_{i=1}^{4} P_{i}^{F M S}(t), w_{4}=10
\end{array},\right.
$$

and the instantaneous availability of the FMS in different demand shown as follows:

As shown in Figure 8, the instantaneous availability of the FMS for different demands can be obtained according to the Lz-transform method, which helps the manufacturers to design the best production plan in line with customer's order requirements. It should be noted that the availability results are calculated under the constraints that setup changes occur randomly and the machine synchronization rule. Consequently, the Lz-transform can not only be used to evaluate the output of products manufactured by FMS but also to evaluate the system availability effectively in accordance with the changes in product demand.

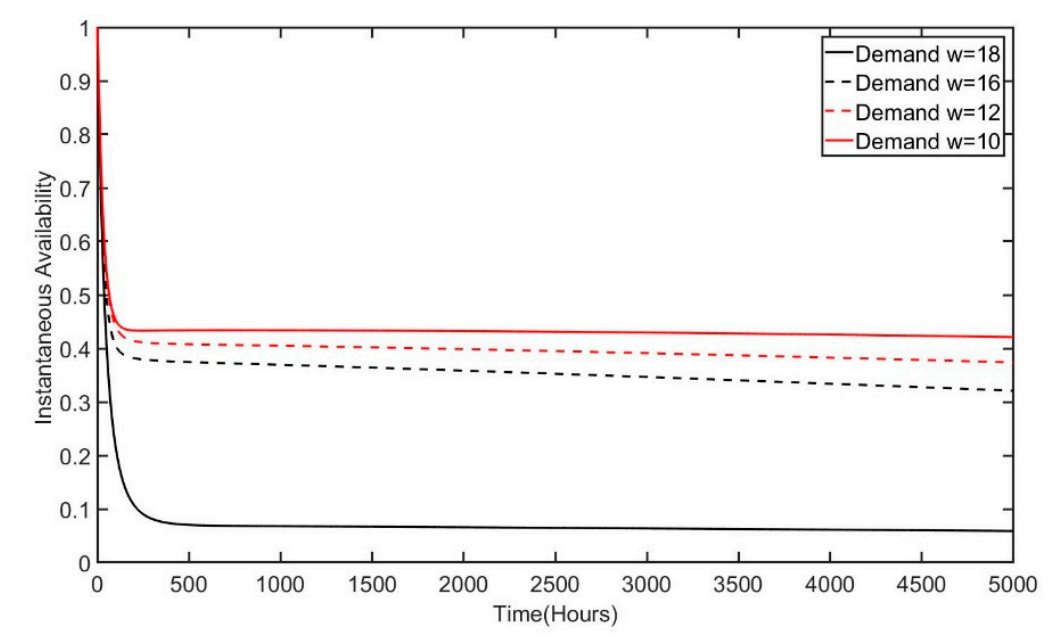

Figure 8. Instantaneous availability the FMS under different demands.

\section{Conclusions}

The setup preparation activities existing in the actual production of CNC machine tools were included to establish a novel multistate transitions process of CNC machine tools in the current paper, which easily demonstrated the production flexibility of the machines as well as the FMS. The following availability and FCI assessment of failure states conducted by the Markov reward approach based on the new model are given. Availability results are helpful to decision making about selecting the initial processing product for the CNC machine tools. Moreover, in the early operation phase of machine tools, the FCI analysis results show that the random setup command contributes more to the system downtime than the failures occurring in the system. Therefore, it is irrational to change settings 
frequently in the early stages of the machine's life cycle, which could greatly reduce the equipment utilization ratio.

An FMS demonstration case built in this paper was based on the new CNC machine tools multistate transitions process. Then the Lz-transform was introduced to the evaluation of output performance and availability for the multistate FMS. The calculation process demonstrated that the Lz-transform is well formalized and suitable for application in performance analysis of an FMS. Moreover, the method allows a dramatic increase in computation efficiency in comparison with the straightforward Markov method that otherwise would have required the building of and solution for a model with "states explosion". The instantaneous availability of the FMS for different order demands can be obtained according to the proposed method, which helps the manufacturers to design the best production plan in line with customers' order requirements.

Future work concerning the FMS should be more practical by relaxing the assumption that the production setup activities occur randomly while more consideration should be given to the actual production scheduling. Extending the research based on the proposed methodology to estimate the impact of the setups and intermediate buffer on the performance of FMS is of interest. Additionally, the optimal maintenance planning study of FMS is significant, which should combine the system availability constraint and customer demand.

Author Contributions: Conceptualization, W.Z., I.F., I.B., and J.K.; Methodology, I.F., L.K., and A.L.; Formal analysis, W.Z.; Writing-original draft preparation, W.Z. and B.C.; Writing-review and editing, I.F., I.B., J.K., L.K., and A.L.; Supervision, G.S. and H.-G.H.; Funding acquisition, B.C.

Funding: This research was funded by the Jilin Provincial Department of Science and Technology Development Project, grant number 20170101212JC. The APC was funded by the Fundamental Research Funds for the Central Universities, JLU.

Acknowledgments: Currently, the stay of Wenbin Zeng in the Institute of Energy Conversion Technology is funded by China Scholarship Council (CSC).

Conflicts of Interest: The author(s) declared no potential conflicts of interest with respect to the research, authorship and/or publication of this article.

\section{References}

1. Mishra, R.S.; Saxena, H.; Madan, A.K. Performance measurement of flexible manufacturing system: A case study. Int. J. Res. Eng. Innov. 2019, 3, 230-234.

2. Roblek, V.; Meško, M.; Krapež, A. A Complex View of Industry 4.0. Sage Open 2016, 6, 2158244016653987. [CrossRef]

3. Pisching, M.A.; Junqueira, F.; Santos Filho, D.J.; Miyagi, P.E. Service composition in the cloud-based manufacturing focused on the industry 4.0. In Doctoral Conference on Computing, Electrical and Industrial Systems; Springer: Cham, Switzerland, 2015; pp. 65-72.

4. Thames, L.; Schaefer, D. Software-defined Cloud Manufacturing for Industry 4.0. Procedia Cirp 2016, 52, 12-17. [CrossRef]

5. He, C.; Zhang, S.Y.; Qiu, L.M.; Liu, X.J.; Wang, Z.L. Assembly Tolerance Design Based on Skin Model Shapes Considering Processing Feature Degradation. Appl. Sci. 2019, 9, 3216. [CrossRef]

6. Browne, J.; Dubois, D.; Rathmill, K.; Sethi, P.; Steke, K.E. Classification of flexible manufacturing systems. FMS Mag. 1984, 14-27.

7. Chang, G.A.; Peterson, W.R. Modeling and Analysis of Flexible Manufacturing Systems: A Simulation Study. In Proceedings of the 2015 ASEE Annual Conference \& Exposition, Seattle, WA, USA, 14-17 June 2015.

8. Groover, M.P. Automated Assembly System, Automation, Production Systems, and Computer-Integrated Manufacturing; Prentice Hall Press: Upper Saddle River, NJ, USA, 2007.

9. Manu, G.; Vijay Kumar, M.; Nagesh, H.; Jagadeesh, D.; Gowtham, M.B. Flexible Manufacturing System (FMS): A Review. Int. J. Mech. Prod. Eng. Res. Dev. 2018, 8, 323-336.

10. Rao, R.V.; Parnichkun, M. Flexible Manufacturing System Selection Using a Combinatorial Mathematics Based Decision Making Method. Int. J. Prod. Res. 2009, 47, 6981-6998. [CrossRef] 
11. Chatterjee, P.; Chakraborty, S. FMS Selection Using Preference Ranking Method: A Comparative Study. Int. J. Ind. Eng. Comput. 2014, 5, 315-338.

12. Taha, Z.; Rostam, S. A hybrid fuzzy AHP-PROMETHEE decision support system for machine tool selection in flexible manufacturing cell. J. Intell. Manuf. 2012, 23, 2137-2149. [CrossRef]

13. Singholi, A.; Ali, M.; Sharma, C. Evaluating the Effect of Machine and Routing Flexibility on Flexible Manufacturing System Performance. Int. J. Serv. Oper. Manag. 2013, 16, 240-261. [CrossRef]

14. Dosdogru, A.T.; Gocken, M.; Geyik, F. Integration of Genetic Algorithm and Monte Carlo to Analyze the Effect of Routing Flexibility. Int. J. Adv. Manuf. Technol. 2015, 81, 1379-1389. [CrossRef]

15. Jain, V.; Raj, T. Modeling and Analysis of FMS Performance Variables by ISM, SEM and GTMA Approach. Int. J. Prod. Econ. 2016, 171, 84-96. [CrossRef]

16. Gyulai, D.; Pfeiffer, A.; Monostori, L. Robust Production Planning and Control for Multi-Stage Systems with Flexible Final Assembly Lines. Int. J. Prod. Res. 2017, 55, 3657-3673. [CrossRef]

17. Rybicka, J.; Tiwari, A.; Enticott, S. Testing a Flexible Manufacturing System Facility Production Capacity through Discrete Event Simulation: Automotive Case Study. Int. J. Mech. Aerosp. Ind. Mechatron. Manuf. Eng. 2016, 10, 668-672.

18. Das, K.; Lashkari, R.; Sengupta, S. Reliability consideration in the design and analysis of cellular manufacturing systems. Int. J. Prod. Econ. 2007, 105, 243-262. [CrossRef]

19. Elleuch, M.; Bacha, H.B.; Masmoudi, F.; Maalej, A.Y. Analysis of Cellular Manufacturing Systems in the Presence of Machine Breakdowns. J. Manuf. Technol. Manag. 2008, 19, 235-252. [CrossRef]

20. Loganathan, M.K.; Girish, K.; Gandhi, O.P. Availability evaluation of manufacturing systems using Semi-Markov model. Int. J. Comput. Integr. Manuf. 2016, 29, 720-735. [CrossRef]

21. Tan, B.; Gershwin, S.B. Analysis of a General Markovian Two-Stage Continuous-Flow Production System with a Finite Buffer. Int. J. Prod. Econ. 2009, 120, 327-339. [CrossRef]

22. Koulamas, C.P. A stochastic model for a machining cell with tool failure and tool replacement considerations. Comput. Oper. Res. 1992, 19, 717-729. [CrossRef]

23. Savsar, M. Reliability analysis of a flexible manufacturing cell. Reliab. Eng. Syst. Saf. 2000, 67, 147-152. [CrossRef]

24. Duan, J.G.; Xie, N.; LI, L.H. Modelling and evaluation of multi-state reliability of repairable non-series manufacturing system with finite buffers. Adv. Mech. Eng. 2019, 11, 1687814019855483. [CrossRef]

25. Chen, K.S.; Yu, C.M.; Hus, T.H.; Cai, S.R.; Chiou, K.C. A Model for Evaluating the Performance of the Bearing Manufacturing Process. Appl. Sci. 2019, 9, 3105. [CrossRef]

26. Kim, J.; Hwangbo, H. Sensor-Based Real-Time Detection in Vulcanization Control Using Machine Learning and Pattern Clustering. Sensors 2018, 18, 3123. [CrossRef] [PubMed]

27. Viswanadham, N.; Ram, R. Composite Performance-Dependability Analysis of Cellular Manufacturing Systems. IEEE Trans. Robot. Autom. 1994, 10, 245-258. [CrossRef]

28. Zakarian, A.; Kusiak, A. Modeling Manufacturing Dependability. IEEE Trans. Robot. Autom. 1997, 13, 161-168. [CrossRef]

29. Das, K.; Lashkari, R.S.; Sengupta, S. Machine Reliability and Preventive Maintenance Planning for Cellular Manufacturing Systems. Eur. J. Oper. Res. 2007, 183, 163-180. [CrossRef]

30. Gupta, P.; Lal, A.K.; Sharma, R.K.; Singh, J. Analysis of Reliability and Availability of Serial Processes of Plastic-Pipe Manufacturing Plant. Int. J. Qual. Reliab. Manag. 2007, 24, 404-419. [CrossRef]

31. Hagen, W.F. Effects of a Reliability Program on Machine Tool Reliability. In Proceedings of the Annual Reliability and Maintainability Symposium, Newport Beach, CA, USA, 23-26 January 2006; pp. 481-485.

32. Lisnianski, A.; Frenkel, I.; Khvatskin, L. On sensitivity analysis of aging multi-state system by using Lz-transform. Reliab. Eng. Syst. Saf. 2017, 166, 99-108. [CrossRef]

33. Bolvashenkov, I.; Herzog, H.G.; Frenkel, I.; Khvatskin, L.; Lisnianski, A. Safety-Critical Electrical Drives: Topologies, Reliability, Performance; Springer: Berlin/Heidelberg, Germany, 2018.

34. Lisnianski, A.; Frenkel, I.; Khvatskin, L. On Birnbaum importance assessment for aging multi-state system under minimal repair by using the Lz-transform method. Reliab. Eng. Syst. Saf. 2015, 142, 258-266. [CrossRef]

35. Frenkel, I.; Lisnianski, A.; Khvatskin, L. Availability Assessment for Aging Refrigeration System by Using Lz-transform. Math. Sci. Res. J. 2012, 5, 33-43. 
36. Lisnianski, A.; Frenkel, I.; Ding, Y. Multi-state System Reliability Analysis and Optimization for Engineers and Industrial Managers; Springer: London, UK, 2010.

37. Lisnianski, A. Lz-Transform for a Discrete-State Continuous-Time Markov Process and its Applications to Multi-State System Reliability. In Recent Advances in System Reliability. Signatures, Multi-State Systems and Statistical Inference; Lisnianski, A., Frenkel, I., Eds.; Springer: London, UK, 2012; pp. 79-95.

38. Ushakov, I. A Universal Generating Function. Sov. J. Comput. Syst. Sci. 1986, 24, 37-49.

39. Zhang, F.; Jardine, A.K.S. Optimal maintenance models with minimal repair, periodic overhaul and complete renewal. IIE Trans. 1998, 30, 1109-1119. [CrossRef]

40. Xie, M.; Dai, Y.S.; Poh, K.L. Computing System Reliability: Models and Analysis; Kluwer Academic/Plenum Publishers: Boston, MA, USA; London, UK, 2004.

41. Howard, R. Dynamic Programming and Markov Processes; MIT Press: Cambridge, MA, USA, 1960.

42. Mine, H.; Osaki, S. Markovian Decision Processes; Elsevier: New York, NY, USA, 1970.

43. Trivedi, K.; Bobbio, A. Reliability and Availability Engineering. Modeling, Analysis, and Applications; Cambridge University Press: Cambridge, UK, 2017.

44. Lisnianski, A.; Frenkel, I. Non-homogeneous Markov reward model for aging multi-state system under minimal repair. Int. J. Perform. Eng. 2009, 5, 303-312.

45. Senanayake, C.D.; Subramaniam, V. Analysis of a two-stage, flexible production system with unreliable machines, finite buffers and non-negligible setups. Flex. Serv. Manuf. J. 2013, 25, 414-442. [CrossRef]

46. Mahmood, K.; Karaulova, T.; Otto, T.; Shevtshenko, E. Performance Analysis of a Flexible Manufacturing System (FMS). Procedia Cirp 2017, 63, 424-429. [CrossRef]

47. Zeng, W.B.; Shen, G.X.; Bolvashenkov, I.; Frenkel, I.; Kammermann, J.; Herzog, H.G. On Non-homogeneous Markov Reward Model to Availability and Importance Analysis for CNC Machine Tools. In Proceedings of the Third International Symposium on Stochastic Models in Reliability Engineering, Life Sciences and Operations Management (SMRLO), Beijing, China, 29-31 May 2019.

48. Toledano, S.; Gartsman, I.; Avitan, G.; Frenkel, I.; Khvatskin, L. On Markov Reward Approach to Failure Criticality Importance Assessment for Aging Multi-state System. In Proceedings of the 2016 Second International Symposium on Stochastic Models in Reliability Engineering, Life Science and Operations Management (SMRLO), Beer-Sheva, Israel, 15-18 February 2016; pp. 375-379.

49. Wang, W.; Loman, J.; Vassiliou, P. Reliability Importance of Components in a Complex System. In Proceedings of the Annual Symposium Reliability and Maintainability, 2004-RAMS, Los Angeles, CA, USA, 26-29 January 2004.

(C) 2019 by the authors. Licensee MDPI, Basel, Switzerland. This article is an open access article distributed under the terms and conditions of the Creative Commons Attribution (CC BY) license (http://creativecommons.org/licenses/by/4.0/). 